\title{
Comprehensive analysis of clinical, pathological, and genomic characteristics of follicular helper T-cell derived lymphomas
}

Sang Eun Yoon ${ }^{1}$, Junhun $\mathrm{Cho}^{2}$, Yeon Jeong Kim³ , Young Hyeh Ko${ }^{2}$, Woong-Yang Park ${ }^{3}$, Seok Jin Kim ${ }^{1}$ and Won Seog $\mathrm{Kim}^{1 *}$ (D)

\begin{abstract}
Background: The 2016 World Health Organization (WHO) classification introduced nodal lymphomas of T follicular helper (Tfh) cell origin, such as angioimmunoblastic T-cell lymphoma (AITL), follicular peripheral T-cell lymphoma (F-PTCL), and nodal peripheral T-cell lymphoma with T follicular helper phenotype (nodal PTCL with TFH phenotype). However, the accurate incidence rate and clinical characteristics of F-PTCL and nodal PTCL with TFH are unstudied.

Methods: Between February 2012 to June 2020, a total of 207 cases diagnosed with nodal lymphomas of T follicular helper (Tfh) cell origin and PTCL-NOS were reviewed for clinical and histopathologic data. PTCL-NOS was defined to not correlate to any of the specific entities of mature T cell lymphoma in the WHO 2016 classification. We attempted to classify PTCL-GATA3 and PTCL-TBX21 by IHC staining. Target gene analysis was performed on a few patients with sufficient blood and tissue samples additionally.
\end{abstract}

Results: Among 207 patients, 111 patients (53.6\%) had AITL, 67 patients (32.4\%) had PTCL-NOS, 19 patients (9.2\%) had F-PTCL, and 10 patients (4.8\%) had nodal PTCL with TFH phenotype. We re-defined and analyzed F-PTCL and nodal PTCL with TFH phenotype into other TFH lymphomas. AITL ( $N=101 / 111,91.0 \%)$ was found to have a higher frequency of stage III/IV cancers compared to other TFH lymphomas ( $N=22 / 29,75.0 \%)$ and PTCL-NOS $(N=53 / 67,79.1 \%$; $p$-value $=0.03$ ). The OS of AITL and other TFH lymphomas was similarly superior to PTCL-NOS ( $p$-value $=0.02)$. AITL and other TFH lymphomas showed the TBX21 subtype more commonly than the GATA3 subtype. Mutations related to the RAS family (RHOA) and those related to epigenetic regulators (IDH2, DNMT3A, and TET2) were shown mainly in AITL and other TFH lymphomas.

Conclusions: Other TFH lymphomas appear to be a rare disease entity around one-quarter in nodal lymphomas of T follicular helper (Tfh) cell origin. Their less aggressive clinical feature than we did not expect is utterly different from PTCL-NOS and AITL. On the other hand, other TFH lymphomas share some characteristics, such as the cell of origin, a more common TBX21 subtype, and genetic variation such as RAS family mutation and epigenetic regulators, with AITL.

Keywords: Peripheral T-cell lymphoma-NOS, Angioimmunoblastic T-cell lymphoma, Follicular peripheral T-cell lymphoma, Nodal peripheral T-cell lymphoma with T follicular helper phenotype

\footnotetext{
*Correspondence: wskimsmc@skku.edu

1 Division of Hematology-Oncology, Department of Medicine, Samsung Medical Center, Sungkyunkwan University School of Medicine, 81, Irwon-ro, Gangnam-Gu, Seoul 06351, Korea

Full list of author information is available at the end of the article
}

\section{Background}

Follicular helper T-cells (Tfh cells) are a subset of CD4+ T-cells that play two roles, serving as memory cells in the T-cell zone of lymphoid organs and effector T cells

c) The Author(s) 2021. This article is licensed under a Creative Commons Attribution 4.0 International License, which permits use, sharing, adaptation, distribution and reproduction in any medium or format, as long as you give appropriate credit to the original author(s) and the source, provide a link to the Creative Commons licence, and indicate if changes were made. The images or other third party material in this article are included in the article's Creative Commons licence, unless indicated otherwise in a credit line to the material. If material is not included in the article's Creative Commons licence and your intended use is not permitted by statutory regulation or exceeds the permitted use, you will need to obtain permission directly from the copyright holder. To view a copy of this licence, visit http://creativeco mmons.org/licenses/by/4.0/. The Creative Commons Public Domain Dedication waiver (http://creativecommons.org/publicdomain/ zero/1.0/) applies to the data made available in this article, unless otherwise stated in a credit line to the data. 
in areas of inflammation, depending on CCR7 homing chemokine expression $[1,2]$. Normal Tfh cells, usually found in the germinal center of lymph nodes, produce IL21 and IL4; these stimulate B cells, suppress regulatory $\mathrm{T}$ cell differentiation, and lead to the proliferation of $\mathrm{Tfh}$ cells [3]. Previous studies have reported that the onset of Tfh-origin lymphoma in this normal immune process is caused by RHOAG17V mutation, which is involved in cell motility, adhesion, and cell-cell interactions [4-7].

The 2016 World Health Organization (WHO) classification introduced nodal lymphomas of $\mathrm{T}$ follicular helper (TFH) cell origin through the expression of at least 2 or 3 TFH markers, including CD279/PD1, CD10, BCL6, CXCL13, ICOS, SAP, and CXCR5 [8]. It has been categorized into three different subtypes under the same umbrella, including angioimmunoblastic T-cell lymphoma (AITL), follicular peripheral T-cell lymphoma (F-PTCL), and nodal peripheral T-cell lymphoma with $\mathrm{T}$ follicular helper phenotype (nodal PTCL with TFH phenotype) [9]. Furthermore, PTCL-NOS was recently defined as excluding F-PTCL and nodal PTCL with TFH.

These three subtypes, AITL, F-PTCL, and nodal PTCL with TFH phenotype, were reported to share molecular abnormalities related to the same origin of Tfh cells [10-12]. Actually, the well-known mutation of AITL, such as RHOA GTPase, TET2, DNMT3A, and IDH2, was also reported in F-PTCL and nodal PTCL with TFH [13]. However, clinical features and therapeutic outcomes of these follicular helper T-cell-derived lymphomas have never been compared because these are relatively rare disease entities, especially F-PTCL and nodal PTCL with TFH. Furthermore, as the F-PTCL and nodal PTCL with TFH were established from the previous provisional disease entities, there are little data about their clinical behaviors and treatment outcomes. Therefore, we performed this comprehensive investigation of follicular helper T-cell derived lymphomas, including clinical, pathological, and molecular features of the Tfh cell origin lymphomas, and compared their treatment response and survival outcome with that of PTCL-NOS.

\section{Methods}

\section{Study data collection and pathology review}

By reviewing the all tissue sample at the diagnosis by hematopathologists from 2012, we collected 207 cases of AITL, F-PTCL, nodal PTCL with TFH, and PTCLNOS from Samsung Medical Center among lymphoma cohort studies (the first cohort, NCT\#01877109; the second cohort, NCT\#03117036). Given that other TFH lymphomas' rarity, we re-defined and analyzed F-PTCL and nodal PTCL with TFH phenotype into other TFH lymphomas. This study was approved by the Institutional Review Board of Samsung Medical Center (approval number. 2016-11-040-019). Written informed consent was obtained from each patient before study enrollment. It was conducted in accordance with the ethical principles of the Declaration of Helsinki and the Korea Good Clinical Practice guidelines.

Based on medical records, we gathered clinical information, including sex, age, complete blood count (CBC), direct antiglobulin test, indirect antiglobulin test, haptoglobin, immunoglobulin GAM, lactate dehydrogenase (LDH), beta-2 microglobulin (B2M), Eastern Cooperative Oncology Group (ECOG) performance status, bone marrow involvement, organomegaly, International Prognostic Index (IPI) [14], and Ann Arbor stage. We further collected the most used front-line chemotherapies, response rate, and survival outcomes according to subtype. The last patient registration was finished in December 2019, and the cut-off date for this study was June 2020.

\section{Pathologic review of T-cell lymphomas}

All cases were reviewed by hematopathologists to differentiate AITL, F-PTCL, nodal PTCL with TFH, and PTCL-NOS according to the WHO 2016 criteria [2]. Three different subtypes under the same umbrella with the $\mathrm{T}$-follicular helper ( $\mathrm{Tfh}$ ) phenotype were sorted through the presence of follicular dendritic cell (FDC) meshwork and follicular growth pattern by staining with CD21 and Tfh markers (CD4, PD-1, CXCL13, BCL6, and CD10). AITL was defined as the Tfh phenotype lymphoma with partial or total effacement of lymph node architecture and expanded FDC meshwork. Also, cases showing specific presentations in tissue samples such as, prominent high endothelial venules (HEVs) in paracortex, polymorphous inflammatory backgrounds containing histiocytes, plasma cells, and eosinophils, and expansion of follicular dendritic cell (FDC) meshwork, were classified as AITL. F-PTCL was characterized by a follicular growth pattern of Tfh-phenotype tumor cells lacking interfollicular involvement. The Tfh phenotype lymphoma without both FDC meshwork expansion and follicular growth pattern was classified as nodal PTCL with TFH (Additional file 1: Fig. S1a).

In addition, we performed immunohistochemistry (IHC) stains for T-bet, CXCR3, GATA3, and CCR4 on Formalin-Fixed Paraffin-Embedded (FFPE) tissue specimens obtained at the time of diagnosis. We tried to classify PTCL-GATA3 (T-helper 2 like origin) and PTCLTBX21(T-helper 1 like origin) among follicular helper through these results T-cell origin lymphomas. PTCLGATA3 type was defined when T-bet or CXCR3 were expressed more than $20 \%$, vice versa PTCL-TBX21 type was defined as when GATA3 or CCR4 were expressed more than $50 \%$ without expression of T-bet and CXCR3 
(Additional file 1: Fig S1b, Additional file 2: Table S1) [15-17].

\section{Blood sampling and targeted deep sequencing}

Among 207 patients, target gene analysis was performed on 69 patients with sufficient blood at the time of examination for diagnosis. Whole blood samples were collected in Cell-Free DNA BCT tubes (Streck Inc., Omaha, NE, USA). After separation of plasma in the initial centrifugation, agranulocytes were separated by Ficoll gradient centrifugation and the granulocytes were separated from the bottom lymphocytes using RBC lysis buffer (Qiagen, Santa Clarita, CA, USA). Genomic DNA (gDNA) was isolated from granulocytes using a QIAamp DNA mini kit (Qiagen, Santa Clarita, CA, USA). Plasma DNA was obtained from 2 to $5 \mathrm{~mL}$ of plasma using a QIAamp Circulating Nucleic Acid Kit (Qiagen). The PBLs and plasma DNA libraries were created using a KAPA Hyper Prep Kit (Kapa Biosystems, Woburn, MA, USA) as described previously. In addition, capture baits for 66 genes selected from a 426 gene panel were customized and used for sequencing of cfDNA and their matched normal samples. After preprocessing, we identified somatic point mutations based on the previously reported iDES-enhanced CAPP-Seq with a minor modification [18]. The filtering steps to identify the variants were summarized as previously described [19].

\section{Tissue sample preparation}

Target gene analysis was performed on tissues of 27 patients who collected plasma. An AllPrep DNA/RNA Mini Kit (Qiagen) was used to purify gDNA from formalin-fixed paraffin-embedded (FFPE) samples. The tumor biopsy sample libraries were constructed using the SureSelect XT reagent kit, HSQ (Agilent Technologies) according to the manufacturer's instructions, and hybrid selection was performed using customized baits targeting 426 lymphoma-related genes. The filtering steps to identify the variants are summarized as previously described [20].

\section{Statistical analysis}

Descriptive statistics were determined as proportions and medians, and the intergroup comparisons for categorical variables were assessed by the $X 2$ or Fisher's exact test. The Kaplan-Meier method was used to evaluate progression-free survival (PFS) and overall survival (OS). PFS time was estimated as the time from diagnosis to the date of disease progression or death related to any cause. OS time was assessed as the time from diagnosis to the date of death or the last date of follow-up. All data were analyzed using the Statistical Package for Social Sciences software, version 24.0 (IBM Corp, Armonk, NY, USA).

\section{Results}

Characteristics description according to each subtype

Among 207 patients, AITL was the most common diagnosis $(\mathrm{N}=111,53.6 \%)$. Sixty-seven patients (32.4\%) were diagnosed with PTCL-NOS, and the minority of patients had confirmed F-PTCL $(\mathrm{N}=19,9.2 \%)$ or nodal PTCL with TFH phenotype $(\mathrm{N}=10,4.8 \%)$. We have presented the clinical characteristics according to subtypes, such as AITL, other TFH lymphomas, and PTCL-NOS, in Table 1. Half of the patients with AITL $(\mathrm{N}=66 / 111$, $61.6 \%)$ and other TFH lymphomas $(\mathrm{N}=15 / 29,51.7 \%)$ were over 60 years old, compared to those with PTCLNOS ( $p$-value $=0.05)$. Stage III/IV disease $(N=176 / 207$, $85.0 \%)$ at diagnosis was more common than stage I/II disease $(\mathrm{N}=31 / 207,15 \%)$. In particular, AITL $(\mathrm{N}=101 / 111$, 91.0\%) was found to have a higher frequency of stage III/IV disease at diagnosis than other TFH lymphomas $(\mathrm{N}=22 / 29,75.0 \%)$ and PTCL-NOS $(\mathrm{N}=53 / 67,79.1 \%$; $p$-value $=0.03)$. The percentage of patients evaluated with high-intermediate or high IPI groups (IPI $\geq 2$ ) was $67.6 \%$ in AITL $(\mathrm{N}=75 / 111), 37.9 \%$ in other TFH lymphomas $(\mathrm{N}=11 / 29)$, and $50.7 \%$ in PTCL-NOS $(\mathrm{N}=34 / 67$; $p$-value $=0.01)$. Additionally, we tried to assess potential prognostic factors among a total of 207 patients with AITL, other TFH lymphomas, or PTCL-NOS. In both univariate and multivariate analysis, male, ECOG performance status $\geq 2$, and thrombocytopenia were especially associated with inferior overall survival for the patients (Additional file 2: Table S2).

Splenomegaly was more common in AITL $(\mathrm{N}=53 / 111,47.7 \%)$ compared to other TFH lymphomas $(\mathrm{N}=6 / 29,20.7 \%)$ and PTCL-NOS $(\mathrm{N}=24 / 67,35.8 \%$; $p$-value $=0.02)$. Bone marrow involvement was demonstrated in 46 patients with AITL (41.4\%), nine patients with other TFH lymphomas (31.0\%) and 18 patients with PTCL-NOS (27.7\%; $p$-value $=0.17)$. Elevated LDH was more common in AITL and PTCL-NOS compared to other TFH lymphomas $(65.1 \%$ vs. $56.7 \%$. vs. $34.5 \%$; $p$-value $=0.01)$. Anemia was confirmed in 43 patients (20.8\%), of which nine were hemolytic anemia reported only in patients diagnosed with AITL. Furthermore, hyper-paraproteinemia was mainly observed in AITL, and less frequently in other TFH lymphomas and PTCL. At the time of diagnosis, $38 \%$ of patients $(\mathrm{N}=80 / 207)$ had B-symptoms, and the frequency of B-symptoms was the highest in AITL $(\mathrm{N}=56 / 111,50.5 \%)$; only $27.6 \%$ of patients presented with B-symptoms in other TFH lymphomas $(\mathrm{N}=8 / 29)$. Thrombocytopenia $(18.3 \%$ vs. $0 \%$ vs. $23.9 \%$; $p$-value $=0.01)$, elevated B2M ( $58.6 \%$ vs. $24.1 \%$ vs. $52.2 \%$; $p$-value $=0.00$ ), and serum EBV detection rate $(40.5 \%$ vs. $31.0 \%$ vs. $11.9 \%$; $p$-value $=0.00)$ were more common in AITL compared to other TFH lymphomas or PTCL-NOS. 
Table 1 Comparison of clinical characteristics according to subtype

\begin{tabular}{|c|c|c|c|c|c|}
\hline Patients characteristics & Total & AITL & Other TFH lymphomas & PTCL-NOS & $p$-value \\
\hline$N(\%)$ & 207 & $111(53.6)$ & $29(14.0)$ & $67(32.4)$ & \\
\hline Age $\geq 60$ years & $99(47.8)$ & $66(61.1)$ & $15(51.7)$ & $27(40.3)$ & 0.05 \\
\hline \multicolumn{6}{|l|}{ Sex } \\
\hline Male & $132(63.8)$ & $64(57.7)$ & $16(55.2)$ & $52(77.6)$ & \multirow[t]{2}{*}{0.01} \\
\hline Female & $75(36.2)$ & $47(42.3)$ & $13(44.8)$ & $15(22.4)$ & \\
\hline \multicolumn{6}{|l|}{ ECOG } \\
\hline $0-1$ & $182(87.9)$ & $95(85.6)$ & $24(82.2)$ & $63(94.0)$ & \multirow[t]{2}{*}{0.15} \\
\hline$>2$ & $25(12.1)$ & $16(14.4)$ & $5(17.2)$ & $4(6.0)$ & \\
\hline \multicolumn{6}{|l|}{ Stage } \\
\hline$|/| \mid$ & $31(15.0)$ & $10(9.0)$ & $7(24.1)$ & $14(20.9)$ & \multirow[t]{2}{*}{0.03} \\
\hline III/IV & $176(85.0)$ & $101(91.0)$ & $22(75.9)$ & $53(79.1)$ & \\
\hline \multicolumn{6}{|l|}{$|\mathrm{P}|$} \\
\hline$<2$ & $87(42.0)$ & $36(32.4)$ & $18(62.1)$ & $33(49.3)$ & \multirow[t]{2}{*}{0.01} \\
\hline$\geq 2$ & $120(58.0)$ & 75 (67.6) & $11(37.9)$ & $34(50.7)$ & \\
\hline B-symptom, presence & $80(38.6)$ & $56(50.5)$ & $8(27.6)$ & $16(23.9)$ & 0.00 \\
\hline $\mathrm{Hb}<10 \mathrm{~g} / \mathrm{dL})$ & $43(20.8)$ & $31(28.4)$ & $2(6.9)$ & $10(14.9)$ & 0.01 \\
\hline Hemolytic anemia & $9(4.3)$ & $9(8.1)$ & 0 & 0 & 0.00 \\
\hline Platelet $<100 \mathrm{k}$ & $36(17.4)$ & $20(18.3)$ & 0 & $16(23.9)$ & 0.01 \\
\hline Elevated LDH & $119(57.5)$ & $71(65.1)$ & $10(34.5)$ & $38(56.7)$ & 0.01 \\
\hline Elevated B2M & $107(51.7)$ & $65(58.6)$ & $7(24.1)$ & $35(52.2)$ & 0.00 \\
\hline $\lg G \geq 1600 \mathrm{mg} / \mathrm{dL}$ & $44(21.3)$ & $33(29.7)$ & $2(6.9)$ & $9(13.4)$ & 0.00 \\
\hline $\lg A \geq 400 \mathrm{mg} / \mathrm{dL}$ & $23(11.1)$ & $16(14.4)$ & $2(6.9)$ & $5(7.5)$ & 0.00 \\
\hline $\operatorname{lgM} \geq 230 \mathrm{mg} / \mathrm{dL}$ & $30(14.5)$ & $29(26.1)$ & $1(3.4)$ & $0(0.0)$ & 0.00 \\
\hline Serum EBV, positive & $62(30.0)$ & $45(40.5)$ & $9(31.0)$ & $8(11.9)$ & 0.00 \\
\hline Splenomegaly & $83(40.1)$ & $53(47.7)$ & $6(20.7)$ & $24(35.8)$ & 0.02 \\
\hline BM involvement & 73 (35.3) & $46(41.4)$ & $9(31.0)$ & $18(27.7)$ & 0.17 \\
\hline
\end{tabular}

ECOG Eastern Cooperative Oncology Group, LDH lactate dehydrogenase, B2M beta-2 microglobulin, EBV Epstein-Barr virus; BM, bone marrow

\section{Treatment outcomes and survival outcomes related to each subtype}

Of 205 patients who received front-line chemotherapy, the majority of patients $(\mathrm{N}=149 / 204,72.0 \%)$ received CHOP-like (CHOP21, CHOP14, mini-CHOP) or CHOEP-like (EPOCH, DA-EPOCH, CHOEP) chemotherapy. Fewer patients received ICE (Ifosfamide, Mesna, Carboplatin, Etoposide $(\mathrm{N}=25 / 204,12.1 \%)$ or gemcitabine-based chemotherapy $(\mathrm{N}=10 / 204,4.8 \%)$ as a front-line strategy. Three patients did not have an opportunity to receive chemotherapy due to fatal disease progression before starting treatment. Among 149 patients who received combined anthracycline chemotherapies, patients diagnosed with AITL achieved an ORR of $76.8 \%$ $(\mathrm{N}=63 / 82)$ and a CR of $70.7 \%(\mathrm{~N}=58 / 82)$, while those diagnosed with PTCL-NOS obtained an ORR of $63.6 \%$ $(\mathrm{N}=31 / 49)$ and a $\mathrm{CR}$ of $44.9 \%(\mathrm{~N}=22 / 49)$. Notably, patients diagnosed with other TFH lymphomas obtained a slightly higher ORR of $94.4 \%(\mathrm{~N}=17 / 18)$ and $\mathrm{CR}$ of 83.3\% $(\mathrm{N}=15 / 18)$ compared to those diagnosed with AITL and PTCL-NOS (Fig. 1).
When we compared PFS and OS according to AITL, PTCL-NOS, and other TFH lymphomas, we found that patients diagnosed with AITL and other TFH lymphomas had similar PFS (17.7 months, 95\% CI 10.5-24.9; 23.8 months, 95\% CI 6.3-41.3; 9.1 months, 95\% CI 5.4$12.8, p$-value $=0.04)$ and OS (60.0 months, 95\% CI $55.8-$ 64.2; 60.0 months, 95\% CI 12.4-107.6; 12.6 months, 95\% CI 18.5-28.7, $p$-value $=0.02)$, and these were superior compared to PTCL-NOS (Fig. 2).

\section{Analysis of molecular studies and immunohistochemistry analysis}

Under the current guidelines, we performed IHC staining (T-bet, CXCR3, GATA3, CCR4) to estimate the proportion of TBX21 subtype and GATA3 subtype in 66 patients who had appropriate FFPE samples (Fig. 4a). Among 41 patients diagnosed with AITL, TBX21 was identified in 31 patients (75.6\%), and GATA3 was described in 9 patients (22.0\%). In other TFH lymphomas $(\mathrm{N}=11)$, the TBX21 subtype $(\mathrm{N}=10 / 11,90.9 \%)$ was more common than the GATA3 
Others : 20 patients $(9.7 \%)$

\begin{tabular}{|c|c|c|c|}
\hline Subtypes & No. patients & ORR (N, \%) & CR (\%) \\
\hline AITL & 7 & $5(71.4)$ & $5(71.4)$ \\
\hline $\begin{array}{l}\text { Other THF } \\
\text { lymphomas }\end{array}$ & 4 & $2(50.0)$ & $2(50.0)$ \\
\hline PTCL-NOS & 9 & $5(55.6)$ & $4(44.4)$ \\
\hline \multicolumn{4}{|c|}{ Gemcitabine based : 10 patients (4.8\%) } \\
\hline Subtypes & No. patients & $\operatorname{ORR}(\mathrm{N}, \%)$ & CR (\%) \\
\hline AITL & 5 & $3(60.0)$ & $2(40.0)$ \\
\hline $\begin{array}{l}\text { Other THF } \\
\text { lymphomas }\end{array}$ & 4 & $2(50.0)$ & $2(50.0)$ \\
\hline PTCL-NOS & 1 & $1(100.0)$ & $0(0.0)$ \\
\hline Total & 10 & $6(60.0 \%)$ & $4(40.0 \%)$ \\
\hline
\end{tabular}

ICE : 25 patients (12.1\%)

\begin{tabular}{|c|c|c|c|}
\hline Subtypes & No. patients & ORR (N, \%) & CR (\%) \\
\hline AITL & 15 & $10(66.7)$ & $6(40.0)$ \\
\hline $\begin{array}{c}\text { Other THF } \\
\text { lymphomas }\end{array}$ & 3 & $2(66.7)$ & $2(66.7)$ \\
\hline PTCL-NOS & 7 & $5(71.4)$ & $3(42.9)$ \\
\hline Total & 25 & $\begin{array}{c}17 \\
(68.0 \%)\end{array}$ & $\begin{array}{c}11 \\
(44.0 \%)\end{array}$ \\
\hline
\end{tabular}

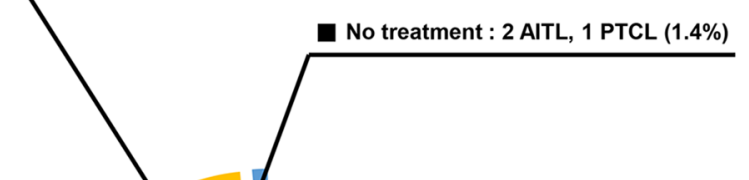

Fig. 1 Overview of response rate according to front-line chemotherapy

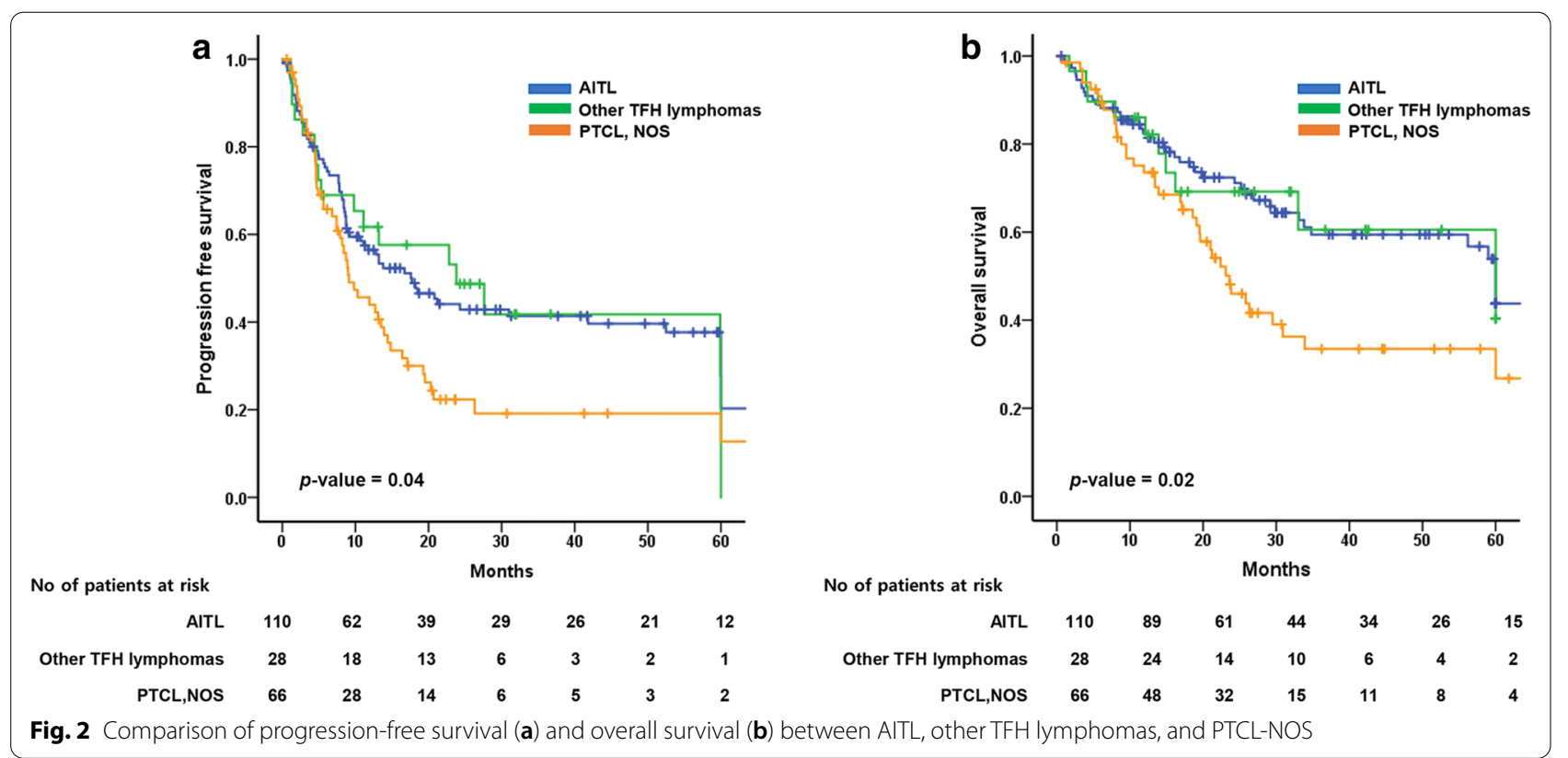

subtype $(\mathrm{N}=1 / 11,9.1 \%)$. However, the proportion of TBX21 subtype $(\mathrm{N}=8 / 15,53.3 \%)$ and GATA3 subtype $(\mathrm{N}=7 / 15,46.7 \%)$ identified in PTCL-NOS showed no difference (Fig. 3b). However, we could not prove the significant difference of OS between patients with TBX21 and GATA3 ( $p$-value $=0.15$ ) from our findings
(Additional file 1: Fig. S2). Comparing the staining results, the TBX21 subtype is more common in AITL and other TFH lymphomas than the GATA3 subtype. However, staining results did not demonstrate a significant difference between other TFH lymphomas and PTCL-NOS $(p$-value $=0.084)$, other TFH lymphomas 


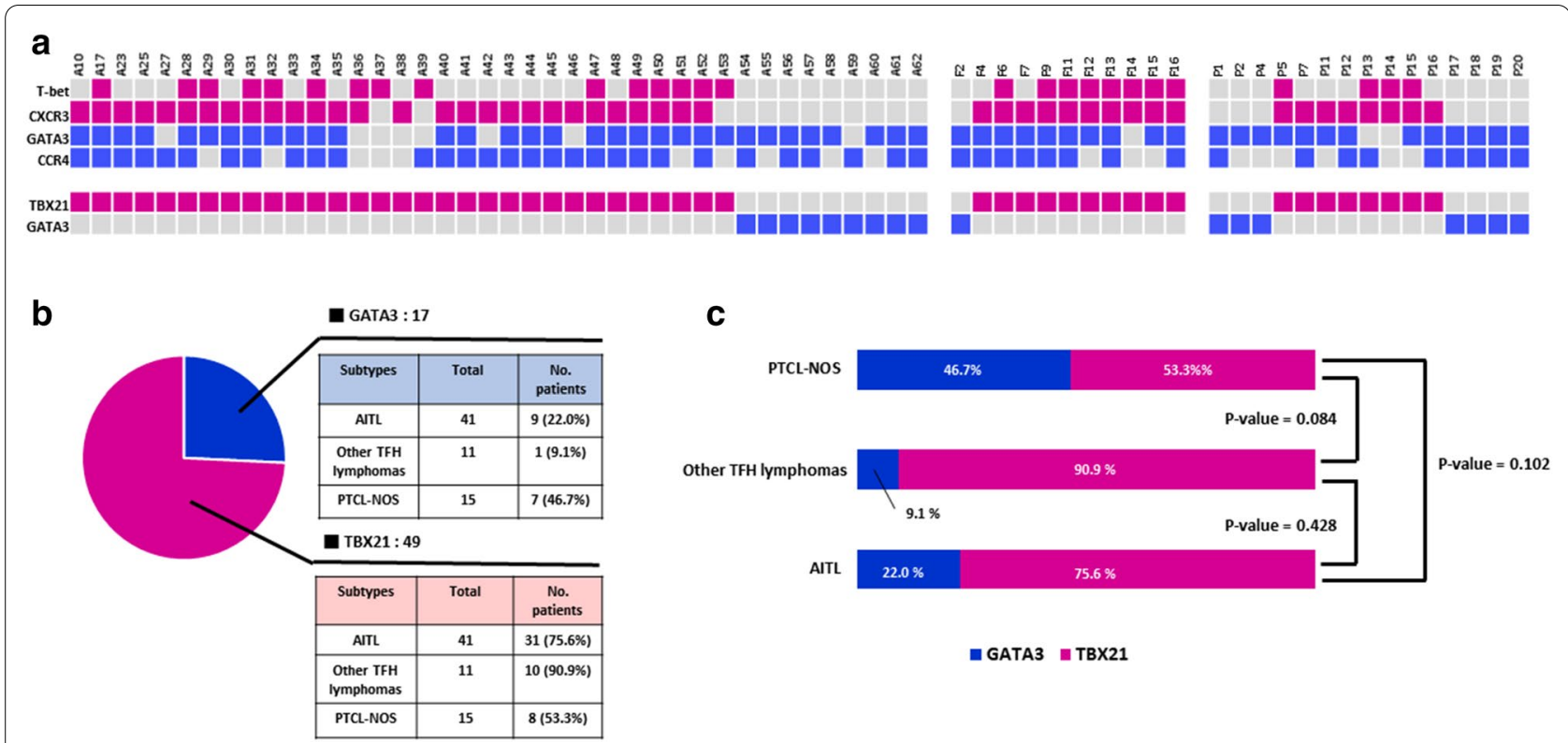

Fig. 3 Overview of TBX21 and GATA3 staining results in AITL, other TFH lymphomas, and PTCL-NOS (a), the number of samples available for TBX21 and GATA3 staining (b), comparison of TBX21 and GATA3 expression among AITL, other TFH lymphomas, and PTCL-NOS (c)

and AITL ( $p$-value $=0.428)$, or PTCL-NOS and AITL $(p$-value $=0.102$; Fig. $3 c)$.

In addition, we tried to describe genetic aberrations among AITL, other TFH lymphomas, and PTCL-NOS using blood and tissue samples collected at diagnosis through next-generation sequencing (NGS). Notably, mutations related to the RAS family $(R H O A)$ and those related to epigenetic regulators (IDH2, DNMT3A, and TET2) were found mainly in AITL and other TFH lymphomas. There was no pattern of multiple $R H O A$ presentations, and the most common RHOA G17V mutation was 20 cases, followed by 3 cases of $R H O A$ T19I. However, mutations belonging to the TCR pathway, transcription factors, and tumor suppressors were found equally in AITL, other TFH lymphomas, and PTCL-NOS (Fig. 4). Finally, we attempted to confirm the association between IHC results and genetic

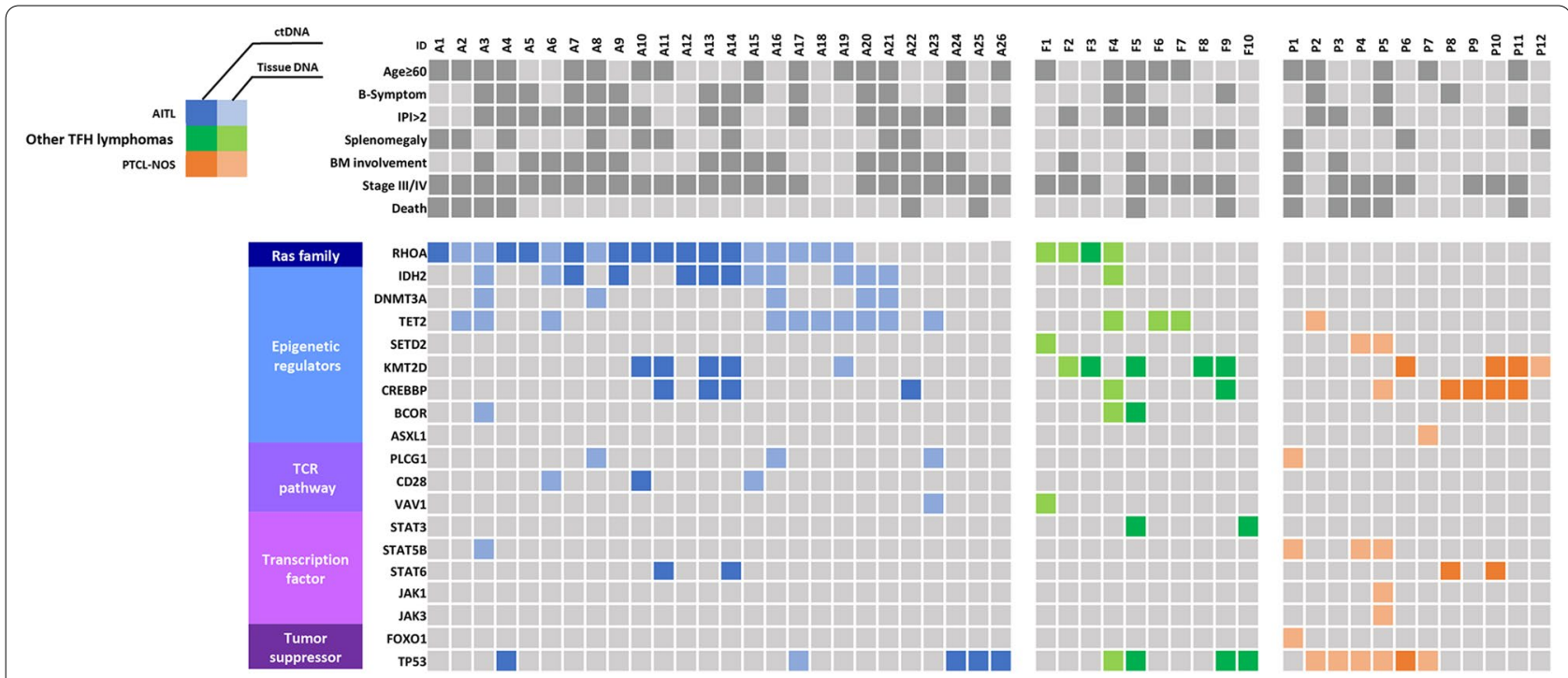

Fig. 4 Overview of genetic expression and matched clinical information 
alterations, but this was challenging due to the shortage of samples (Additional file 1: Fig. S3).

\section{Discussion}

According to 2016 WHO classification, PTCL-NOS with a T-follicular helper (TFH) cell phenotype was redefined into three subtypes, AITL, F-PTCL, and nodal PTCL with TFH, based on clinicopathologic features, immunohistochemistry (IHC), and typical genetic features [9]. However, due to the rarity of other TFH lymphomas, including F-PTCL and nodal PTCL with TFH, most studies retrospectively reported a pathologic review or molecular studies $[13,21,22]$. Thus, we could not precisely identify the clinical characteristics and disease progression patterns of other TFH lymphomas separated from AITL and PTCL-NOS. According to our retrospective data, other TFH lymphomas seems to present with less aggressive clinical features and has a favorable treatment response compared to AITL and PTCL-NOS. Moreover, the overall survival rate of other TFH lymphomas was similar to AITL and had better outcomes than PTCL-NOS. Although other TFH lymphomas was classified as PTCL-NOS with TFH cells, it expressed mainly T-bet and CXCR3, like PTCL-TBX21 (Th1 cell origin) [23]. As reported in other studies, the representative genetic alterations to induce a robust $\mathrm{T}$ follicular helper phenotype (RHOA, IDH2, and DNMT3A) of AITL also appeared in other TFH lymphomas [24]. However, other mutation profiles that play an essential role in T-cell receptor (TCR) signaling, transcription factors, and tumor suppressors were shared evenly between AITL, other TFH lymphomas, and PTCL-NOS [25].

PTCL-NOS includes all T-cell lymphomas with ambiguous pathological, clinical, and biological characteristics within the 2008 and 2016 WHO classifications. The International T-cell Lymphoma Project conducted a multicenter registry study to estimate the incidence of each subtype of PTCL worldwide. A diagnosis of PTCL-NOS was checked in $25.9 \%$, and AITL was confirmed in $18.5 \%$ [26]. Furthermore, an Asian registry study also reported that PTCL-NOS represented $20.8 \%$ and AITL represented $24.7 \%$ of all cases [27]. Although there are differences in reported rates by each region, the incidence rate is around $20 \%$. Since previous studies on the incidence rate of PTCLs were conducted based on the 2008 WHO classification, there was no mention of F-PTCL and nodal PTCL with TFH, which has started to attract attention based on the new 2016 WHO classification. We were able to re-review the pathology of 207 cases with PTCLNOS and AITL and found 29 (14\%) other TFH lymphomas cases through this study. Although our retrospective study could not represent all T-cell lymphoma entities, other TFH lymphomas is predicted to account for a very small number of T-cell lymphoma classifications, less than about $10 \%$ of PTCL-NOS. As such, the incidence rate of other TFH lymphomas is meager, and the search process is complex and challenging, requiring more careful attention during diagnosis.

Several studies have shown that survival outcomes of patients with PTCL-NOS are usually inferior compared to those with AITL. Several studies reported a 5-year overall survival (OS) rate of AITL patients ranging from 33 to $48 \%$ [28] and PTCL-NOS patients around 30\% retrospectively $[26,29]$. However, we found that the survival of PTCL-NOS excluding other TFH lymphomas were worse than that of AITL, while other TFH lymphomas and AITL had similar survival rates. Moreover, the CR rate of PTCL patients with standard anthracycline-based therapy showed ranges from 40 to $60 \%$, according to previous studies [30, 31]. In this study, other TFH lymphomas demonstrated an excellent CR rate (about 80\%) compared to PTCL-NOS (about 40\%). Therefore, in previous studies, the PTCL-NOS is more likely to have included other TFH lymphomas, which have a better prognosis, and this likely overestimates the chemotherapy response rate and survival outcomes of PTCL-NOS. Therefore, PTCL-NOS needs to be classified in detail to obtain accurate survival and response rates in future registry studies or clinical studies targeting PTCLs.

PTCL-GATA3 exhibited T-helper 2 cell differentiation and presented genomic complexity with frequent loss or mutation of tumor suppressor genes targeting the $C D K 2 A / B-T P 53$, and PTEN-PIK3 pathways. PTCLTBX21 manifested T-helper 1 cell differentiation and showed more mutations of cytotoxic effector genes and epigenetic regulator genes [32]. Heavican et al. showed that the mutation profile of PTCL-NOS with a TFH cell phenotype consisted of TET2, DNMT3A, and RHOA, which are common in AITL [33]. In our study, we also observed that other TFH lymphomas and AITL were mainly classified as PTCL-TBX21 based on IHC staining; these also exhibited RHOA, IDH2, and DNMT3A, which are included in the category of epigenetic modulators (Fig. 4). Thus, we suggest that other TFH lymphomas and AITL might show a better response rate to treatment with epigenetic modifiers and hypomethylating agents than PTCL-NOS. Paola et al. already conducted a retrospective study to show the efficacy of histone deacetylase inhibitors (HDACi) in PTCL-NOS with TFH versus non-TFH phenotypes. The response rate to HDACi in PTCL-NOS with a TFH cell phenotype was about twice as high as that of the non-TFH phenotype $(56.5 \%$ vs. $29.4 \%, p$-value $=0.0035$ ) [34]. Therefore, our data suggest that new target agents under development or research for AITL might also be promising treatment strategies in other TFH lymphomas. 
Interestingly, hypergammaglobulinemia was shown more in AITL, less in other TFH lymphomas [8]. In histopathologic findings, AITL is characterized by the proliferation of high endothelial venules and mixed inflammatory cell infiltration, which is not prominent in other TFH lymphomas [35, 36]. Hypergammaglobulinemia is caused by increased immunoglobulin production of polyclonal plasma cells, and this phenomenon can be considered a result of specific immunogenic stimulation of AITL. In other words, it can be assumed that the immunogenicity of tumor cells is one of the essential factors that make the clinical and pathological differences between AITL and other TFH lymphomas.

Through target sequencing research previously performed, we designed a customized panel of 66 genes targeting somatic mutations for various NHLs. Based on the research of longitudinal plasma samples using this panel, it was found that the sensitivity $(88.0 \%)$ and specificity ( $>99 \%)$ of the panel to detect somatic mutation were higher than that of tumor biopsies [19]. Same as the previous method, DNA was extracted and analyzed from separating each plasma, and blood cells, followed by germline DNA was filtered using DNA information from blood cells. Thus, it was available to consider the remained mutation as somatic mutations, not germline mutations. Thus, although blood and tissue samples were not obtained simultaneously in our study, we were able to compare the somatic mutation profile features between AITL, other TFH lymphomas, and PTCL-NOS based on the result that ctDNA well reflects the characteristics of the tumor tissue. Another limitation of our genetic data is that DMNT2A and TET2 have a discrepancy between ctDNA and tissue samples. The first reason is the panel for tissue (425 genes) included more somatic mutation genes than that for ctDNA (66 genes). The second reason is $D M N T 2 A$, and TET2 representing the clonal hematopoiesis of indeterminate potential (CHIP) did not include when we generated the ctDNA panel because these genes originated from neutrophils and generally filtered out due to low VAF. Although data related to somatic mutations did not represent the characteristics of AITL, other TFH lymphomas, and PTCL-NOS comprehensively, our findings were sufficient to show their tendency.

Given the rarity of the F-PTCL and nodal PTCL with $\mathrm{TFH}$, we were unable to separate the two and analyze them as different diseases according to our findings. Although we integrated and analyzed the two diseases by other TFH lymphomas, we were able to observe the relative proportion, clinical features, and molecular characteristics of other TFH lymphomas. Consequently, it seems that other TFH lymphomas is a unique disease entity completely different from PTCLNOS and AITL; however, it shares some characteristics like the cell of origin and genetic variation with AITL. Even though other TFH lymphomas is classified as a separate entity, PTCL-NOS remains a basket term inclusive of all unspecified entities within the 2016 WHO classification. Thus, further studies are continually needed to identify the specific disease characteristics of PTCL-NOS. We emphasize the importance of classifying other TFH lymphomas as a separate disease category from PTCL-NOS in future studies.

\section{Abbreviations}

Tfh: T follicular helper; AITL: Angioimmunoblastic T-cell lymphoma; F-PTCL: Follicular peripheral T-cell lymphoma; nodal PTCL with TFH phenotype, nodal peripheral T-cell lymphoma with T follicular helper phenotype; ECOG: Eastern Cooperative Oncology Group; LDH: Lactate dehydrogenase; B2M: Beta-2 microglobulin; EBV: Epstein-Barr virus; BM: Bone marrow.

\section{Supplementary Information}

The online version contains supplementary material available at https://doi. org/10.1186/s40164-021-00224-3.

Additional file 1: Figure S1. Methods to distinguish the T-follicular helper (Tfh) phenotype by CD21 and Tfh markers (CD4, PD-1, CXCL13, BCL6, and CD10) staining (a), methods to classify PTCL-GATA3 (T-helper 2 like origin) and PTCL-TBX21(T-helper 1 like origin) (b). Figure S2. Comparison of OS between PTCL-GATA3 (T-helper 2 like origin) and PTCL-TBX21(T-helper 1 like origin). Figure S3. Matching results of genetic information and TBX21/ GATA3 staining.

Additional file 2: Table S1. Summary of antibody-related information used for TBX21-PTCL and GATA3-PTCL staining. Table S2. Univariate and multivariate analysis of PFS and OS for a total of 207 patients.

\section{Acknowledgements}

This research was supported by a grant of the Korea Health Technology R\&D Project through the Korea Health Industry Development Institute (KHIDI), funded by the Ministry of Health \& Welfare, Republic of Korea (grant number : HR20C0025).

\section{Authors' contributions}

WSK and SEY conceived and designed the study. SEY, JC, YJK, YHK, WYP, SJK, and WSK study, accrued patients to the study and collected clinical data. SEY and WSK analysed the data and wrote the manuscript. All authors edited the manuscript. All authors read and approved the final manuscript.

\section{Funding}

No companies supported this study.

\section{Availability of data and materials}

All data will become publicly available upon request from the corresponding authors.

\section{Declarations}

Ethics approval and consent to participate

This study was approved by the Institutional Review Board of Samsung Medical Center (Approval Number. 2016-11-040-019). It was conducted in accordance with the ethical principles of the Declaration of Helsinki and the Korea Good Clinical Practice guidelines. 


\section{Consent for publication \\ Not applicable.}

\section{Competing interests}

The authors have no conflicts of interest regarding the publication of this paper.

\section{Author details}

${ }^{1}$ Division of Hematology-Oncology, Department of Medicine, Samsung Medical Center, Sungkyunkwan University School of Medicine, 81, Irwon-ro, Gangnam-Gu, Seoul 06351, Korea. ${ }^{2}$ Department of Pathology, Samsung Medical Center, Sungkyunkwan University School of Medicine, Seoul, Korea.

${ }^{3}$ Samsung Genome Institute, Samsung Medical Center, Seoul, Korea.

\section{Received: 9 March 2021 Accepted: 26 April 2021}

\section{Published online: 14 May 2021}

\section{References}

1. Lanzavecchia A, Sallusto F. Understanding the generation and function of memory T cell subsets. Curr Opin Immunol. 2005;17:326-32.

2. Breitfeld D, Ohl L, Kremmer E, Ellwart J, Sallusto F, Lipp M, et al. Follicular B helper T cells express CXC chemokine receptor 5 , localize to B cell follicles, and support immunoglobulin production. J Exp Med. 2000;192:1545-52.

3. Ahearne MJ, Allchin RL, Fox CP, Wagner SD. Follicular helper T-cells: expanding roles in T-cell lymphoma and targets for treatment. $\mathrm{Br} \mathrm{J}$ Haematol. 2014;166:326-35.

4. Heasman SJ, Carlin LM, Cox S, Ng T, Ridley AJ. Coordinated RhoA signaling at the leading edge and uropod is required for $T$ cell transendothelial migration. J Cell Biol. 2010;190:553-63.

5. Sakata-Yanagimoto M, Enami T, Yoshida K, Shiraishi Y, Ishii R, Miyake Y, et al. Somatic RHOA mutation in angioimmunoblastic T cell lymphoma. Nat Genet. 2014:46:171-5.

6. Yoo HY, Sung MK, Lee SH, Kim S, Lee H, Park S, et al. A recurrent inactivating mutation in RHOA GTPase in angioimmunoblastic T cell lymphoma. Nat Genet. 2014:46:371-5.

7. Fukumoto K, Nguyen TB, Chiba S, Sakata-Yanagimoto M. Review of the biologic and clinical significance of genetic mutations in angioimmunoblastic T-cell lymphoma. Cancer Sci. 2018;109:490-6.

8. Swerdlow SH, Campo E, Harris NL, Jaffe ES, Pileri SA, Stein H, et al. WHO classification of tumours of haematopoietic and lymphoid tissues. 4 th ed. Lyon: International Agency for Research on Cancer; 2008.

9. Swerdlow SH, Campo E, Pileri SA, Harris NL, Stein H, Siebert R, et al. The 2016 revision of the World Health Organization classification of lymphoid neoplasms. Blood. 2016;127:2375-90.

10. Lemonnier F, Couronne L, Parrens M, Jais JP, Travert M, Lamant L, et al. Recurrent TET2 mutations in peripheral T-cell lymphomas correlate with TFH-like features and adverse clinical parameters. Blood. 2012;120:1466-9.

11. Wang C, McKeithan TW, Gong Q, Zhang W, Bouska A, Rosenwald A, et al. IDH2R172 mutations define a unique subgroup of patients with angioimmunoblastic T-cell lymphoma. Blood. 2015;126:1741-52.

12. Palomero T, Couronne L, Khiabanian H, Kim MY, Ambesi-Impiombato A, Perez-Garcia A, et al. Recurrent mutations in epigenetic regulators, RHOA and FYN kinase in peripheral T cell lymphomas. Nat Genet. 2014;46:166-70.

13. Dobay MP, Lemonnier F, Missiaglia E, Bastard C, Vallois D, Jais JP, et al. Integrative clinicopathological and molecular analyses of angioimmunoblastic T-cell lymphoma and other nodal lymphomas of follicular helper T-cell origin. Haematologica. 2017;102:e148-51.

14. International Non-Hodgkin's Lymphoma Prognostic Factors Project. A predictive model for aggressive non-Hodgkin's lymphoma. N Engl J Med. 1993;329:987-94.

15. Iqbal J, Wright G, Wang C, Rosenwald A, Gascoyne RD, Weisenburger DD, et al. Gene expression signatures delineate biological and prognostic subgroups in peripheral T-cell lymphoma. Blood. 2014;123:2915-23.

16. lqbal J, Weisenburger DD, Greiner TC, Vose JM, McKeithan T, Kucuk C, et al. Molecular signatures to improve diagnosis in peripheral T-cell lymphoma and prognostication in angioimmunoblastic T-cell lymphoma. Blood. 2010;115:1026-36.

17. Amador C, Greiner TC, Heavican TB, Smith LM, Galvis KT, Lone W, et al. Reproducing the molecular subclassification of peripheral T-cell lymphoma-NOS by immunohistochemistry. Blood. 2019;134:2159-70.

18. Newman AM, Lovejoy AF, Klass DM, Kurtz DM, Chabon JJ, Scherer F, et al. Integrated digital error suppression for improved detection of circulating tumor DNA. Nat Biotechnol. 2016;34:547-55.

19. Shin SH, Kim YJ, Lee D, Cho D, Ko YH, Cho J, et al. Analysis of circulating tumor DNA by targeted ultra-deep sequencing across various nonHodgkin lymphoma subtypes. Leuk Lymphoma. 2019;60:2237-46.

20. Hyeon J, Lee B, Shin SH, Yoo HY, Kim SJ, Kim WS, et al. Targeted deep sequencing of gastric marginal zone lymphoma identified alterations of TRAF3 and TNFAIP3 that were mutually exclusive for MALT1 rearrangement. Mod Pathol. 2018;31:1418-28.

21. Maura F, Dodero A, Carniti C, Bolli N. Biology of peripheral T cell lymphomas - not otherwise specified: is something finally happening? Pathogenesis. 2016:3:9-18.

22. Timmins MA, Wagner SD, Ahearne MJ. The new biology of PTCL-NOS and AITL: current status and future clinical impact. Br J Haematol. 2020;189:54-66.

23. de Leval L, Gaulard P. Cellular origin of T-cell lymphomas. Blood. 2014;123:2909-10.

24. Watatani Y, Sato Y, Miyoshi H, Sakamoto K, Nishida K, Gion Y, et al. Molecular heterogeneity in peripheral T-cell lymphoma, not otherwise specified revealed by comprehensive genetic profiling. Leukemia. 2019:33:2867-83.

25. Xie C, Li X, Zeng H, Qian W. Molecular insights into pathogenesis and targeted therapy of peripheral T cell lymphoma. Exp Hematol Oncol. 2020;9:30.

26. Vose J, Armitage J, Weisenburger D, International TCLP. International peripheral T-cell and natural killer/T-cell lymphoma study: pathology findings and clinical outcomes. J Clin Oncol. 2008;26:4124-30.

27. Yoon SE, Kim SJ, Yoon DH, Chen TY, Koh Y, Kang KW, et al. 2570 Intercontinental cooperative non-Hodgkin T-cell lymphoma prospective registry study in Asia: ICT study. Ann Oncol. 2020;31:S1343-4.

28. Mourad N, Mounier N, Briere J, Raffoux E, Delmer A, Feller A, et al. Clinical, biologic, and pathologic features in 157 patients with angioimmunoblastic T-cell lymphoma treated within the Groupe d'Etude des Lymphomes de l'Adulte (GELA) trials. Blood. 2008;111:4463-70.

29. Federico M, Bellei M, Marcheselli L, Schwartz M, Manni M, Tarantino V, et al. Peripheral T cell lymphoma, not otherwise specified (PTCL-NOS) A new prognostic model developed by the International T cell Project Network. Br J Haematol. 2018;181:760-9.

30. Gallamini A, Stelitano C, Calvi R, Bellei M, Mattei D, Vitolo U, et al. Peripheral T-cell lymphoma unspecified (PTCL-U): a new prognostic model from a retrospective multicentric clinical study. Blood. 2004;103:2474-9.

31. Weisenburger DD, Savage KJ, Harris NL, Gascoyne RD, Jaffe ES, MacLennan KA, et al. Peripheral T-cell lymphoma, not otherwise specified: a report of 340 cases from the International Peripheral T-cell Lymphoma Project. Blood. 2011;117:3402-8.

32. King C. New insights into the differentiation and function of $T$ follicular helper cells. Nat Rev Immunol. 2009;9:757-66.

33. Heavican TB, Bouska A, Yu J, Lone W, Amador C, Gong Q, et al. Genetic drivers of oncogenic pathways in molecular subgroups of peripheral T-cell lymphoma. Blood. 2019;133:1664-76.

34. Ghione P, Faruque P, Mehta-Shah N, et al. T follicular helper phenotype predicts response to histone deacetylase inhibitors in relapsed/refractory peripheral T-cell lymphoma. Blood Adv. 2020;4:4640-7.

35. Bal M, Gujral S, Gandhi J, Shet T, Epari S, Subramanian PG. Angioimmunoblastic T-cell lymphoma: a critical analysis of clinical, morphologic and immunophenotypic features. Indian J Pathol Microbiol. 2010;53:640-5.

36. Chiba S, Sakata-Yanagimoto M. Advances in understanding of angioimmunoblastic T-cell lymphoma. Leukemia. 2020;34:2592-606.

\section{Publisher's Note}

Springer Nature remains neutral with regard to jurisdictional claims in published maps and institutional affiliations. 